\title{
Genetic and physiological characterization of the OsCem mutant in rice: formation of connected embryos with multiple plumules or multiple radicles
}

\author{
X-c Yang and C-m Hwa \\ Section of Life Sciences, Yangtze University, Jingzhu, China
}

\begin{abstract}
Establishment of the apical-basal axis is a critical event in plant embryogenesis. Two functionally distinct primordia (the plumule and radicle) are meristems that originate from the lower and upper regions, respectively, of the embryo and are arranged along an apical-basal axis. One rice mutant (OsCem), which alters the embryo axis pattern to produce multiple plumules and/or radicles, was characterized. The OsCem mutant plants showed three different phenotypes: a multiple-shoot type, a heart-shaped Siamese embryo having multiple plumules and a common radicle; a multiple-root type, an upside-down Y-shaped Siamese embryo possessing multiple radicles and a common plumule; and a connected twin type, a connected Siamese twin having two sets of plumules and radicles. These diverse phenotypes demonstrated that the upper and lower regions of the embryo
\end{abstract}

Keywords: rice; OsCem mutant; auxin; cleavage polyembryo

\section{Introduction}

Although most morphogenetic events occur in the postembryonic phase of the life cycle, the fundamental body plan in higher plants is established during embryogenesis. Embryogenesis begins with a single-cell zygote, which undergoes a series of cell divisions and differentiation events to establish an apical-basal pattern along the main axis of polarity. As a result, two apical primordia are produced-an embryonic bud and an embryonic root-that have the potential to form meristems. Some studies, based on various embryo mutants in Arabidopsis (Errampalli et al., 1991; Mayer et al., 1991; Jurgens, 2001), indicate that two functionally distinct primordia (meristems) are associated with the localized expression of key genes. Mutations of key genes in Arabidopsis embryogenesis alter the developmental patterns of the embryo to induce extra (or secondary) embryo formation. For example, mutation of the TWN gene in Arabidopsis can alter the developmental program of the suspensor to promote extra (or secondary) embryo formation (Vernon and Meinke, 1994; Zhang and Somerville, 1997). Mutations of the MAPKK kinase gene YODA

Correspondence: Dr C-m Hwa, Section of Life Sciences, Yangtze University, Jingzhu 434025, China.

E-mail: zhimigh@hotmail.com or zhimingh@126.com

Received 31 January 2008; revised 15 April 2008; accepted 18 April 2008; published online 11 June 2008 axis have a similar potential to complete the full spectrum of the developmental program of multiple plumules and radicles, respectively. Genetic analysis and molecular mapping based on simple sequence repeat markers has revealed that a recessive gene was involved in the control of the connected Siamese embryo formation. By using an F2 mapping population derived from a cross between the OsCem mutant and the variety $95-15$, the OsCem locus was mapped primarily to the short arm of chromosome 3 of rice (Oryza sativa) at the interval between markers RM148 $(6.4 \mathrm{cM})$ and RM468 $(7.5 \mathrm{cM})$. Subsequently, the OsCem locus was fine mapped to the interval between markers M5 $(1.6 \mathrm{cM})$ and $\mathrm{M} 6(1.0 \mathrm{cM})$.

Heredity (2008) 101, 239-246; doi:10.1038/hdy.2008.50; published online 11 June 2008

also alters the cell fate of the basal lineage to promote extraembryonic fate during Arabidopsis embryogenesis (Lukowitz et al., 2004).

Rice (Oryza sativa L.), as a model cereal crop, has several advantages for basic biological studies (Shimamoto and Kyozuka, 2002). For example, rice has a highly developed embryo, which contains almost all organs seen in the vegetative phase, such as a shoot apex, 1-3 leaf primordia, a vascular system and a root primordium (Hong et al., 1995). The rice embryo has diverse body axes, including apical-basal, dorsal-ventral and radial patterns, all of which are established during embryogenesis. The plumule and radicle in the rice embryo are arranged along the apical-basal axis of the embryo, whereas the scutellum and coleoptile originate from the dorsal and ventral sides of the embryo, respectively. This stable pattern indicates that functional regionalizations in the rice embryo are regulated by specific gene(s) (Itoh et al., 2005). Although many different mutants in maize (Clark and Sheridan, 1991; Sheridan and Clark, 1993; Heckel et al., 2000) and rice (Nagato et al., 1989; Kitano et al., 1993; Hong et al., 1996; Satoh et al., 1999; Kinae et al., 2005) with abnormal embryonic development profiles have been characterized, rice mutants that produce multiple plumules and/or radicles, and even form a connected twin in the same embryo, have not previously been reported.

Here, we report a novel rice mutant, a connected embryo (OsCem), which altered the developmental 
pattern of the embryo to produce multiple plumules and/or radicles during embryogenesis. Genetic analysis and molecular mapping based on simple sequence repeat (SSR) markers revealed that a single recessive gene was involved in the control of the Siamese embryo formation and the OsCem gene was mapped primarily on the short arm of chromosome 3 of $O$. sativa at the interval between markers M5 $(1.6 \mathrm{cM})$ and M6 $(1.0 \mathrm{cM})$.

\section{Materials and methods}

\section{Plant materials}

Approximately 10 strains of multiple-embryo seedlings during rice breeding have been defined in the past decade, and only 3 of these were inherited after several generations of selection. Some multiple-embryo seedlings originated from two or three different independent embryos in the same ovule. Only one strain, the inbred line B93-12, formed multiple seedlings that originated from the same embryo. This mutant was named connected embryo (OsCem). The OsCem mutant and wild-type plants were grown in the field and were collected as experimental materials; pertinent crosses between the OsCem mutants and wild-type rice strains were performed.

\section{Germination experiments}

Dried seeds of the dehusked mutant were exposed to sunlight 1 day before germination to increase the germination potential. The naked grains were soaked in hot water at $40{ }^{\circ} \mathrm{C}$ for $2 \mathrm{~h}$ and were germinated in wellmoistened filter paper for 5 days at $27^{\circ} \mathrm{C}$ under constant illumination. The germinating seedlings were analyzed under a dissecting microscope.

\section{Histology and whole mounting}

The developmental courses of the wild-type and mutant embryos were examined by the standard paraffin method. For paraffin sectioning, ovules at various developmental stages were fixed in FAA solution (formalin:glacial acetic acid:70\% ethanol, 5:5:90), dehydrated in graded ethanol series and embedded in paraffin. The samples were sectioned at $8 \mu \mathrm{m}$ and stained with hematoxylin.

For the whole mounting method, the mutant seedlings were fixed in ethanol:acetic acid (6:1) for 3-4h at room temperature. After several washes in a graded ethanol series (100, 90, 70 and 50\%), $30 \mathrm{~min}$ for each step, the caryopsis tissues of germinating seedlings were removed under a dissecting microscope. The seedlings without caryopsis were mounted in a mixture solution of chloral hydrate:glycerol:distilled water (8:1:2, w:v:v; Berleth and Jurgens, 1993) and kept for about 6-8h at room temperature. Whole-mount preparations could be stored at room temperature for at least several weeks. The cleared seedlings were visualized using a Leica DMBR microscope with $\times 1$ objective under dark-field illumination. The images were recorded using a Photometrics Sensys CCD camera (KAF0400) and processed using Version 3.5 of the PMIS software package.

\section{Genetic analysis}

Four cross combinations were made to analyze the inheritance of the OsCem locus. The OsCem mutant plants were crossed as a female with one of the male parents of cultivars 87-11 (japonica), DB22 (japonica), Minghui 63 (indica) or 95-15 (indica), respectively. The segregation ratio of phenotypes of the wild type and the mutant in F2 plants of the crosses were analyzed after seed germination. The statistical analysis was performed by the software program Statistix for Windows.

\section{Genetic mapping}

Rice genomic DNA was extracted from the fresh leaves of rice plants using the method previously described by McCouch et al. (1988). The SSR primers for genetic mapping were synthesized according to the sequence data as cited on the Gramene website (http://www.gramene.org). The PCR programs were as follows: 1 cycle at $94{ }^{\circ} \mathrm{C}$ for $5 \mathrm{~min}$; 35 cycles at $94{ }^{\circ} \mathrm{C}$ for $1 \mathrm{~min}$, at $55^{\circ} \mathrm{C}$ for $1 \mathrm{~min}$ and at $72{ }^{\circ} \mathrm{C}$ for $1.5 \mathrm{~min}$; and 1 last cycle at $72^{\circ} \mathrm{C}$ for $5 \mathrm{~min}$. Reactions were carried out in a volume of $25 \mu \mathrm{l}$ containing $1 \mu \mathrm{moll}^{-1}$ of primers, $200 \mu \mathrm{moll}^{-1}$ of $\mathrm{dNTP}$, $5 \mathrm{ng}$ of DNA template, $2 \mathrm{mmoll}^{-1} \mathrm{MgCl}_{2}, 2.5 \mu \mathrm{l} 10 \times$ buffer and $0.6 \mathrm{U}$ of Tag DNA polymerase. The PCR products were separated in a $3 \%$ agarose gel containing $0.15 \mu \mathrm{g} \mathrm{ml}^{-1}$ ethidium bromide in $1 \times$ Tris-Borate-EDTA running buffer. Linkage analyses were performed using the segregation data in the F2 populations by Mapmaker version 3.0 (Lander et al., 1987). Genetic distances between markers were presented in Kosambi centiMorgans $(\mathrm{cM})$.

\section{IAA measurements}

The OsCem mutant and wild-type plants were grown in the field. The embryos used for assessing indole-3-acetic acid (IAA) concentrations were isolated at 3, 4 and 6 DAP (days after pollination), and then transferred to an Eppendorf tube and immersed in liquid nitrogen. The seedlings were frozen at the tenth leaf stage and were saved individually to measure the IAA content of each leaf. IAA was quantified following the procedure of Schmelz et al. (2003). Tissues from the frozen seedlings were analyzed individually and the measurements of IAA in the embryos and seedlings were expressed as a mean $(n=3)$.

\section{Results}

Different phenotype investigations of the OsCem mutant A unique OsCem mutant described here was found in an inbred line, B93-12, of O. sativa that was propagated by selfing, suggesting that the mutation occurred spontaneously in the previous generation. The seedlings of the mutant had different phenotypes according to the number of primary roots and shoots that they possessed (Figure 1) and could be categorized into three groups based on the relationship between the number of primary roots and shoots after germination: (1) Multiple-shoot type: heart-shaped Siamese embryos with multiple plumules and one common radicle (Figure 1d) - the frequency of this type of seedling was about 92.3-95.0\% based on 2 successive years of experiments; (2) Multipleroot type: upside-down Y-shaped Siamese embryos possessed multiple radicles and one common plumule (Figures $1 \mathrm{~b}$ and c) - the frequency of this type of seedling was about $3.1-4.2 \%$ and (3) Connected twin type: connected Siamese embryos had multiple plumules and 

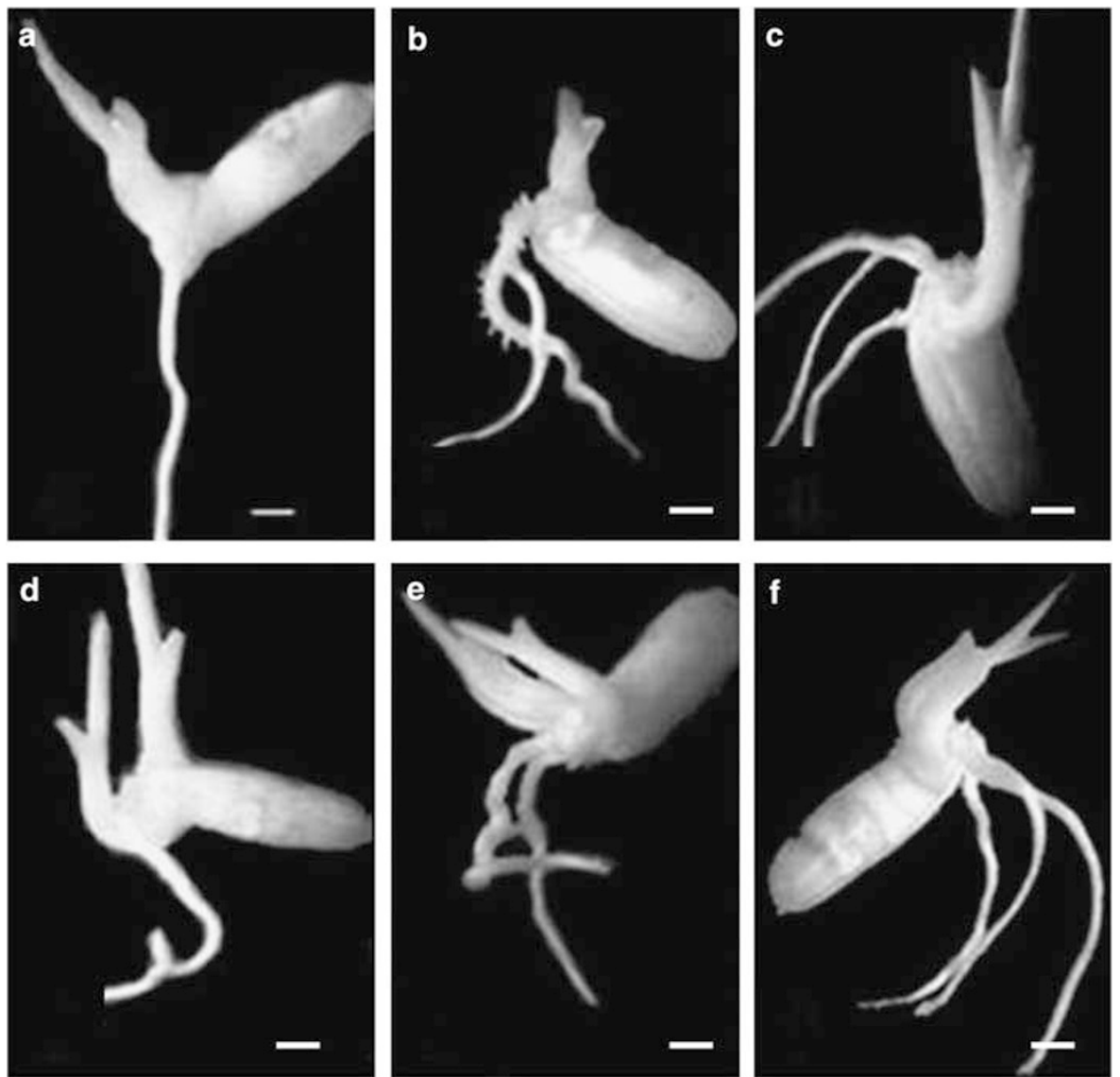

Figure 1 Different phenotypes of seedlings of the OsCem mutant. (a) A wild-type seedling with a shoot and a root. (b) The mutant seedling with two roots and a common shoot. (c) The mutant seedling with three roots and a common shoot. (d) The mutant seedling with two shoots and one common root. (e) The mutant seedling showing a connected twin with two shoots and two roots. (f) The mutant seedling with two shoots and three roots. Bar $=1.2 \mathrm{~mm}$.

multiple radicles (Figures 1e and $\mathrm{f}$ ) - the frequency of this type was about $1.9-3.0 \%$.

To determine the origin of the multiple radicles or plumules, the pattern of embryogenesis of the OsCem mutant was assessed using tissue sectioning. The sectioning data indicated that multiple plumules originated from the ventral and dorsal sides of the same embryo, respectively (Figures $2 \mathrm{a}$ and $\mathrm{b}$ ). The precise origin time of the primordium of the extra (or secondary) plumule was slightly different according to different rachises of the same strain. The initiation of the extra (or secondary) plumule primordium on the dorsal side occurred slightly after that of the normal plumule primordium on the ventral side, in most cases (Figure $2 b$ ), which resulted in the formation of nonequal double seedlings at the early stage of germinating seedlings; the normal and secondary plumules in some embryos sometimes originated at almost the same time, which resulted in the formation of equal-sized double seedlings (Figures $1 \mathrm{~d}$ and 2f). Although both sides of the upper region of the embryo axis formed two plumule primordia, the lower region of the embryo axis developed normally to form a common radicle primordium (Figures $1 \mathrm{~d}$ and $3 \mathrm{~d}$ ). A total of 29 germinating seedlings of the OsCem mutant were examined using the wholemount method, indicating that two plumules of all seedlings shared a common radicle (Figure 3d). Embryos that developed this specific kind of abnormal connected embryo were called heart-shaped Siamese embryos. In spite of the fact that the OsCem mutant had only one root, the double seedlings could develop many normal adventitious roots after germination, suggesting that two shoots of the OsCem mutant seedlings had a similar potential to differentiate normal crown roots.

The upper region of the embryo axis has the potential to develop multiple plumules; the lower region of the embryo axis also has the similar potential to produce multiple radicles. Our results showed that the lower region of the embryo axis can develop extra radicle(s) (Figure 2d), but the frequency of multiple radicles was much lower. From the late-stage globular embryo at about 3 DAP, the lower region of the embryo axis, in which a normal radicle usually forms, expanded to initiate the formation of primordia, such as an extra (or secondary) radicle and a wild-type radicle. The lengths of the two hypocotyls were not equal-one hypocotyl was short and the other was longer, as assessed by observation of longitudinal sections (Figure 2d). To determine whether the extra radicle was linked to the common plumule, germinating seedlings were assessed using the whole-mount method. Fifteen seedlings with two or three primary roots and a common shoot were examined, and the results indicated that both the extra (or secondary) radicle and the normal radicle were 

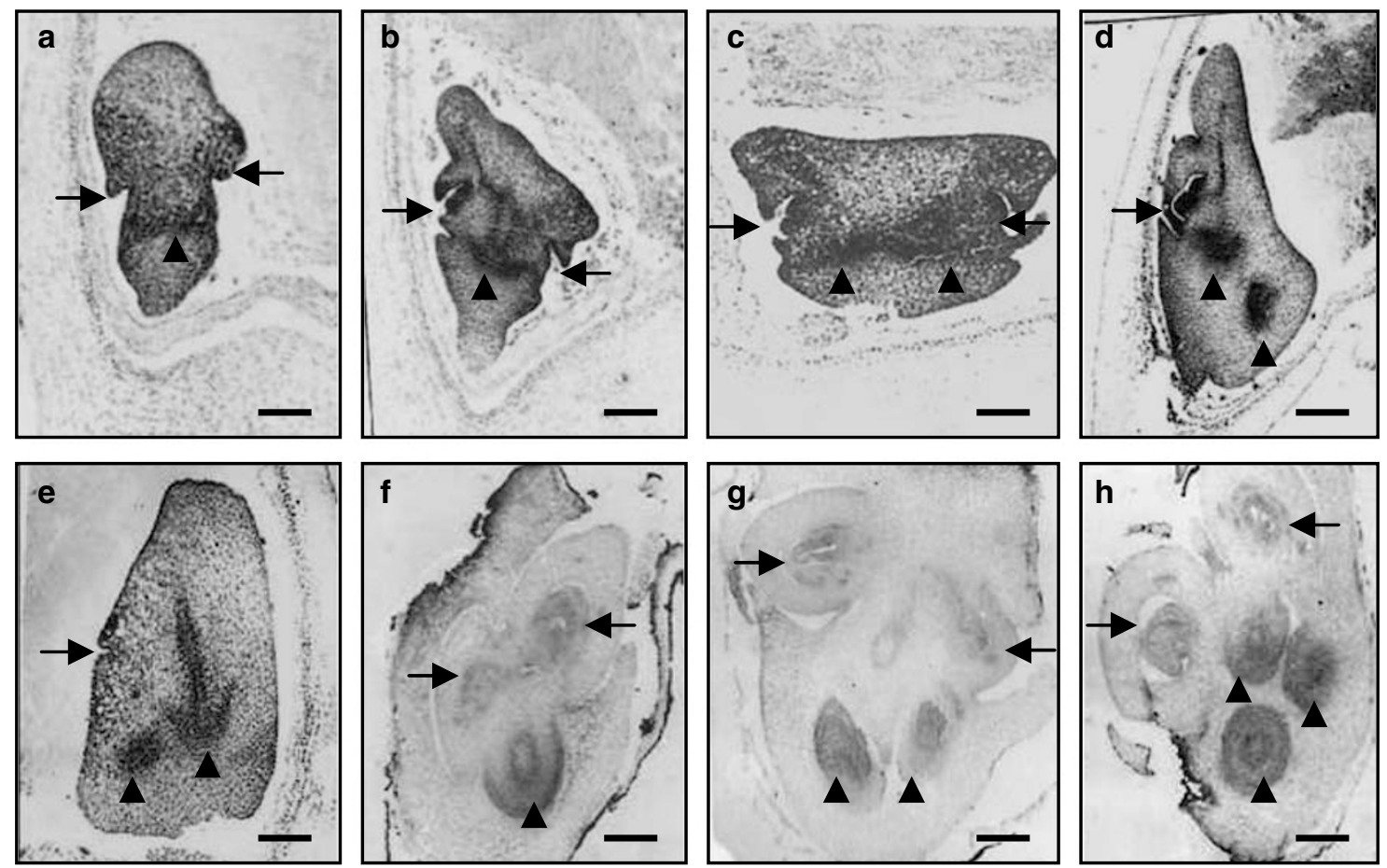

Figure 2 Histological sections of connected embryo development in the OsCem mutant. (a) The differentiation of an extra (or secondary) plumule primordium, a normal plumule primordium and a common radicle primordium. Bar $=50 \mu \mathrm{m}$. (b) The differentiation of two different plumule primordia at opposite sides and a common radicle primordium in the same embryo. Bar $=35 \mu \mathrm{m}$. (c) The differentiation of a Siamese twin. This section shows the expansion at the lower and upper regions of the embryo axis and the origin of two plumules and two radicles in the Siamese twin. Bar $=45 \mu \mathrm{m}$. (d) The origin of an extra radicle primordium. Bar $=30 \mu \mathrm{m}$. (e) The formation of a connected twin. This section shows two radicle primordia and a small part of one plumule primordium and a vascular primordium of another embryo. Bar $=40 \mu \mathrm{m}$. (f) The mature abnormal embryo with two plumules and a common radicle. Bar $=0.2 \mathrm{~mm}$. (g) The mature Siamese twin with two sets of plumules and radicles. Bar $=0.16 \mathrm{~mm}$. (h) The mature Siamese twin with two sets of plumules and radicles, and an extra radicle in the same embryo. Bar $=0.2 \mathrm{~mm}$. Arrows indicate plumule primordium or plumule primordium differentiation; arrowheads indicate radicle primordium or radicle primordium differentiation.

linked to the same plumule by their separated hypocotyls (vascular bundle system; Figures $3 \mathrm{~b}$ and c). This type of abnormal embryo, which develops two or more extra radicles and a common plumule, was called a multipleroot type, or an upside-down, Y-shaped Siamese embryo.

As the lower and upper regions of the embryo axis had a similar potential to initiate formation of multiple radicles or plumules in different embryos, respectively, we thought whether multiple radicles and multiple plumules could be formed in the same embryo. To address the relationship of multiple plumules and multiple radicles in the same embryo, two different phenotypes of the OsCem mutant seedlings in the third group were examined using combined histological sections and the whole-mount method. The results indicated that seedlings of the OsCem mutants in the third group were connected Siamese twins (Figures 2c and e): one shoot was linked to a root via its own hypocotyl (Figure 3e) and another was linked to one or two roots via independent vascular systems (Figures 3e and f). Sectioning data revealed that this Siamese twin originated in two different ways. First, two plumule primordia originated from the dorsal and ventral sides of the same embryo, respectively, and two radicle primordia originated in the enlarged lower region of the embryo axis, resulting in the formation of back-to-back Siamese twins (Figures $2 \mathrm{c}$ and g). Alternatively, two plumule primordia originated from the same ventral side of the embryo and were arranged at upper and lower positions, and the two radicles originated from the expanded lower region of the embryo axis and were positioned side by side (Figures 2e and h).

\section{IAA levels in the OsCem mutant}

Considering that IAA has previously been proposed to play an important role in regulating embryo morphogenesis in Arabidopsis (Friml et al., 2003) and abnormal polyembryo differentiation in wheat embryo culture (Fischer et al., 1997), free IAA concentrations were measured in freshly isolated zygotic OsCem mutant and wild-type embryos at three different developmental stages. The IAA levels of the OsCem mutant and wild-type at the tenth leaf vegetative stages were also assessed.

The IAA concentration in freshly isolated wild-type late globular embryos at 3 DAP was about $14.4 \mathrm{ng} \mathrm{g}^{-1} \mathrm{FW}$ (fresh weight), whereas the IAA level of OsCem mutant late globular embryos was higher (about $20.2 \mathrm{ng} \mathrm{g}^{-1} \mathrm{FW}$ ). The late globular embryo stage is a key stage for organ initiation and tissue differentiation. At the early differentiation stage of the embryos after $4 \mathrm{DAP}$, the IAA level in the OsCem mutant embryos was much higher than that in the wild-type embryos; the IAA concentration in 

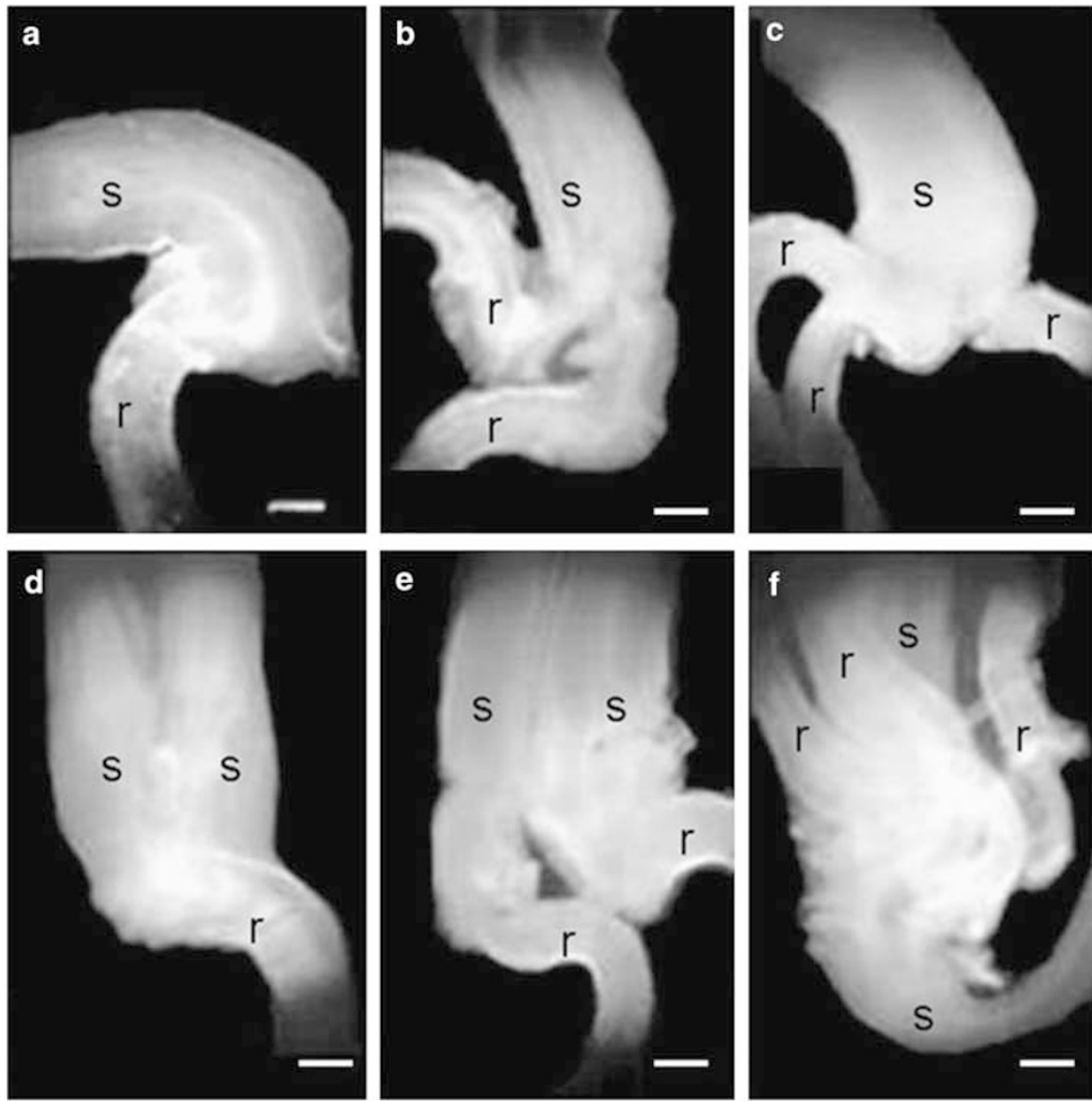

Figure 3 The relationship between multiple plumules and multiple radicles in the mutant seedlings. (a) A wild-type seedling with a shoot and a root. (b) The mutant seedling with one shoot and two roots, one common shoot linked to two roots by two separated hypocotyls. (c) The mutant seedling with one shoot and three roots, one common shoot linked to three roots. (d) The mutant seedling with two shoots and one root, two shoots linked to one common root. (e) The mutant seedling with two shoots and two roots; a connected Siamese twin has an independent vascular system. (f) The mutant seedling with two shoots and three roots, one shoot linked to a root and another common shoot linked to two roots via separated hypocotyls. s, shoot; r, root. Scale bar $=6 \mathrm{~mm}$.

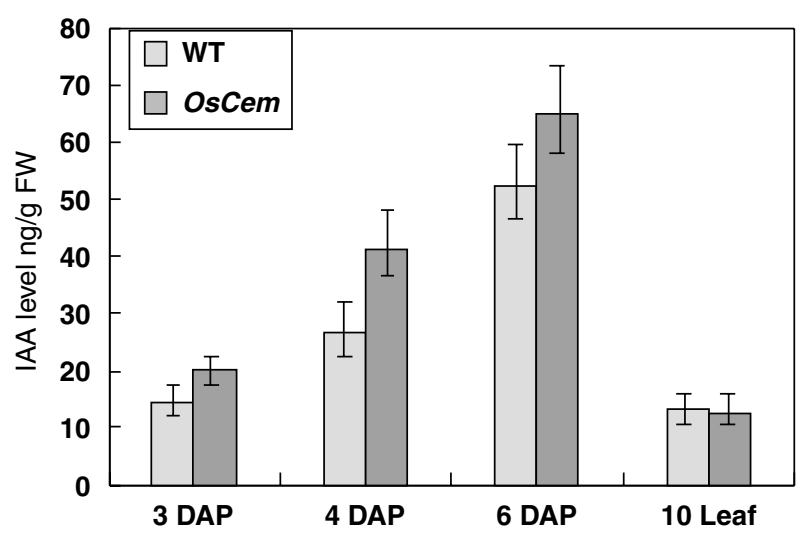

Figure 4 Endogenous indole-3-acetic acid (IAA) levels at different developmental stages of the OsCem mutant and wild-type embryos. The results are the mean of triplicate samples and the bar indicates \pm s.d.

the mutants was $41.5 \mathrm{ng} \mathrm{g}^{-1} \mathrm{FW}$, whereas the IAA level in the wild-type embryos was only $26.8 \mathrm{ng} \mathrm{g}^{-1}$ FW (Figure 4). The IAA concentration of the OsCem mutant-differentiated immature embryos at 6 DAP was $65.3 \mathrm{ng} \mathrm{g}^{-1} \mathrm{FW}$, and the IAA level of the wild-type embryos was $52.4 \mathrm{ng} \mathrm{g}^{-1} \mathrm{FW}$. The different IAA concentrations in OsCem and wild-type embryos were analyzed by analysis of variance, and an F-test was used to evaluate statistically significant differences between the $\mathrm{OsCem}$ and the wild-type data. The results indicated that significant differences $\left(\mathrm{F}=14.2>P_{0.01}=9.33\right)$ in IAA concentration occurred between the OsCem and wildtype embryos. These data suggest that the altered IAA levels in the OsCem and wild-type embryos were, at least partially, linked to abnormal morphogenesis of the OsCem mutant.

Although there were many more differences in the IAA level between the OsCem mutant and the wild type during the formation and differentiation of the embryos, the IAA levels showed no differences at the vegetative stage (OsCem mutant, $13.2 \mathrm{ng} \mathrm{g}^{-1}$ FW; wild type, $12.6 \mathrm{ng} \mathrm{g}^{-1} \mathrm{FW}$ ) (Figure 4). This suggests that the OsCem gene could be expressed specifically in the embryo, but not in the vegetative tissues (including in the leaves).

\section{Genetic analysis and mapping of the OsCem gene} controlling a connected embryo formation

For genetic analysis of the OsCem mutant, four F2 populations were constructed from the crosses between 
the OsCem mutant and each of the four varieties-87-11, 95-15, Minghui 63 and DB22. In the four F1 progeny populations, all seedlings of F1 seeds exhibited wild-type phenotype, suggesting that the mutant trait of the connected embryo was recessive. All seedlings in their F2 progenies had a segregation ratio of 3:1, as predicted by Mendelian inheritance between the wild-type and the mutant plants $\left(X_{C}^{2}<X_{0.05}^{2}=3.84\right.$; Table 1$)$. Therefore, the trait of the connected embryo in the OsCem mutant was controlled by a recessive nuclear gene, $\mathrm{OsCem}$.

To determine the chromosome location of the OsCem gene, 238 SSR markers were used to examine the polymorphisms between the OsCem mutant and the variety 95-15, the two parents of the cross, from which 73 SSR polymorphic markers were selected to investigate a total of 256 F2 plants derived from the cross. The results showed that a SSR marker, RM1004 (located on chromosome 3), was associated with the OsCem phenotype (Figure 5). Following this, more SSR markers that were distal and proximal to RM1004 were used to survey the same F2 population; four markers-RM468, RM1004, RM148 and RM520-were also found to be linked to the mutant trait. There were 8 or 10 recombinants between marker-148 and the OsCem, or marker-468 and the $\mathrm{OsCem}$, respectively. None of the same recombinants

Table 1 Segregation of F2 populations derived from four different crosses

\begin{tabular}{lcccc}
\hline Combination & Wild type & Mutant & Total & $\mathrm{x}^{2}(3: 1)$ \\
\hline OsCem/87-11 & 178 & 57 & 235 & 0.035 \\
OsCem/95-15 & 252 & 78 & 330 & 0.291 \\
OsCem/Minghui63 & 273 & 88 & 361 & 0.045 \\
OsCem/DB22 & 167 & 51 & 218 & 0.220 \\
\hline
\end{tabular}

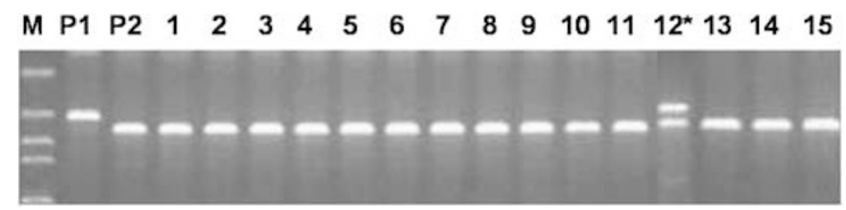

Figure 5 The simple sequence repeat (SSR) marker RM1004 was closely linked to the OsCem locus in primary mapping. P1, the wildtype parent; P2, the OsCem parent; M, DNA ladder; 1-15, the mutant individuals of the F2 population; the asterisk $\left(^{*}\right)$ indicates the recombinant. were found to cover each other between marker-148 and marker-468. Based on the segregation data, the OsCem locus was mapped on chromosome 3 between the molecular markers RM148 and RM468, at distances of 6.4 and $7.5 \mathrm{cM}$, respectively (Figure 6).

For fine mapping of the OsCem gene, we generated a F2 mapping population consisting of more than 4000 plants, derived from the cross between the $\mathrm{OsCem}$ and the 95-15 variety. With the rice genome sequencing completed, more PCR-based markers could be easily designed (http://www.gramene.org/db/searches/ssrtool) according to the available DNA sequences between markers RM148 and RM468. The primers used in these experiments are listed in Table 2. The new-design SSR markers were used to survey more than 913 mutant plants of the F2 population. Subsequently, the OsCem locus was fine mapped to the interval between markers M5 $(1.6 \mathrm{cM})$ and M6 $(1.0 \mathrm{cM})$ (Figure 6).

\section{Discussion}

Multiple-embryo formations have been reported in various species, using either a physiological or a mutagenesis approach. Ferguson et al. (1979) observed that wheat plants produced polyembryos when sprayed immediately after flowering with a herbicide containing 2,4-D. Erdelska and Vidovencova (1992) described the suspensorial embryo and cleavage of the zygotic

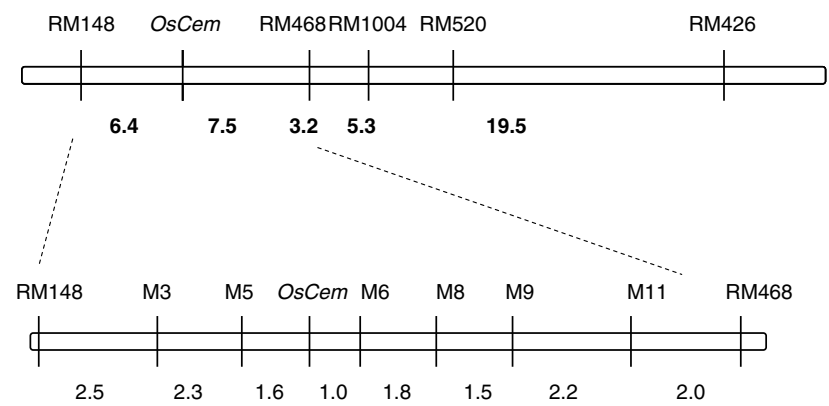

Figure 6 Molecular linkage map of Oryza sativa chromosome 3, showing the location of the OsCem gene. Two simple sequence repeat (SSR) markers, RM148 and RM468, showed close linkage with the OsCem gene on the short arm of chromosome 3 . The linkage distance between the OsCem gene and the markers M5 and M6, was 1.6 and $1.0 \mathrm{cM}$, respectively. The genetic distances between loci were derived by Kosambi mapping function and are shown in centiMorgans (cM).

Table 2 The PCR-based molecular markers designed for fine mapping

\begin{tabular}{|c|c|c|c|}
\hline Markers & Forward primers & Reverse primers & Size \\
\hline M1 & 5'-AGATTGATCTTCTGGAGGTAGC-3' & 5'-AGATTGATCTTCTGGAGGTAGC-3' & 440 \\
\hline M2 & 5'-CCCAAATGTAGTATGCGCCCTAGC-3' & 5'-AGCGCGACGATCTCATCAACG-3' & 587 \\
\hline M3 & 5'-AGCTTTGGTTTCTTGGCTTTGG-3' & 5'-ATTAGCGTTGAACCCAAGTGTGG-3' & 151 \\
\hline M4 & 5'-СТСССАССАТАAСТGСТССТTCG-3' & 5'-AGTGGCAGCGACAGCCATACC-3' & 267 \\
\hline M5 & 5'-GAGATCATCCTGAACAACCACTGC- $3^{\prime}$ & 5'-TGTCСАСССАААСССТСТТТАСС-3' & 194 \\
\hline M6 & 5'-CTCAAGGGAGAAAGCGTAAAGC-3' & 5'-TCTAGTAGAGGCGGAAACAAGG-3' & 277 \\
\hline M7 & 5'-GACGTCAGCGATCTCCACTACG-3' & 5'-CAGCTGTTGCCTGTTGCATAGC-3' & 175 \\
\hline M8 & 5'-TTCGTCGAGTGTTTGATTAGGG-3' & 5'-GTCACCACTGGATAGTCGAATCC-3' & 219 \\
\hline M9 & 5'-AGCAACCGGTGGCCTTCTTGG-3' & 5'-CAGCAGCAGCAGCAAGTGTAAGTGC-3' & 99 \\
\hline M10 & 5'-AATGACGCTGCAGGCTAGATACG-3' & 5'-GTCAGACACGAGATCACGAGAGG-3' & 85 \\
\hline M11 & 5'-TTTGAGCCACCACAAGGACAAGG-3' & 5'-GGCGTGATGTGAAGAAGTGAGACG-3' & 184 \\
\hline
\end{tabular}


proembryo in maize. Cleavage of the zygotic proembryo could also be induced by a 2,4-D treatment. Mutants obtained after wheat or maize pollen irradiation with X-rays produced supernumerary embryos (Morgan and Rappleye, 1951; Unrau and Larter, 1952). All these multiple-embryo formations were induced by a physiological or a chemical approach. The suspensor cells of the twn mutant in Arabidopsis could alter their developmental progress and initiate the formation of a secondary embryo when the apical cell was defective. The altered expression pattern of the TWN gene in the twn2 mutant resulted in the formation of extra (or secondary) embryos (Zhang and Somerville, 1997). Mutation of the FACKEL (FK) gene affected the body organization of the Arabidopsis seedling and resulted in the formation of seedlings with multiple apices or seedlings with multiple roots. FK encodes a sterol C-14 reductase and mediates cell growth and organization during embryonic development (Schrick et al., 2000). Our results show that mutation of the $\mathrm{OsCem}$ gene can induce extra plumule or radicle formation in rice. All these data demonstrate that mutation of key genes controlling embryo development may alter the embryo developmental program and initiate the formation of extra (or secondary) embryo(s).

The phytohormone auxin plays an important role in a wide variety of growth, development and physiological processes (Kepinski, 2006), many of which are dependent on directional auxin polar transport within organs and tissues. The local auxin gradient produced by polar transport represents a common module that operates in the formation of all plant organs, regardless of their mature morphology or developmental origin (Benkova et al., 2003). The formation of root and shoot meristems (Sabatini et al., 1999; Benkova et al., 2003), the establishment of embryo axis patterns (Friml et al., 2003) and the initiation of phyllotaxis primordia (Reinhardt et al., 2003) are related to the auxin gradient. During organ formation in Arabidopsis, the first site of primordia initiation has to be selected and then a new growth axis has to be determined, establishing an auxin gradient with its maximum at the tip (Benkova et al., 2003). The auxin polar transport inhibitors $N$-1-naphthylphthalamic acid and quercetin can induce a number of specific abnormal phenotypes in wheat embryo culture, suggesting that the polar transport of auxin has a determining influence on the differentiation of extra root or shoot meristems (Fischer et al., 1997). Our results also support that the changes in IAA level of embryo may result in the initiation of abnormal embryos in planta. At the late globular embryo stage, there was a difference in IAA concentrations in the wild-type and OsCem mutant embryos. The mutation of the OsCem gene resulted in an increase in IAA concentration at the globular stage, which indicated that the globular embryos of the mutants were preparing new developmental directions or the initiation of a new tissue or organ, and also suggests that the OsCem protein might be related to the auxin signal pathway in embryogenesis. The difference in IAA levels in the wild-type and OsCem mutant embryos was maximum at 4 DAP (Figure 4), suggesting that the formation of the IAA maximum gradient in embryos could result in abnormal morphogenesis. So, the mutation of the OsCem gene may result in the formation of a new IAA gradient, which could be a marker or signal for new tissue or organ initiation during embryo development.

The map-based cloning approach is a method that can be used to isolate the gene of interest based on intensive genetic and physical mapping. The OsCem gene was first anchored in an interval between two molecular markers, M148 and RM468, on chromosome 3. The fine mapping of the OsCem locus suggested that this locus was mapped between two microsatellite markers M5 and M6, with genetic distances of 1.6 and $1.0 \mathrm{cM}$, respectively. Further fine mapping is needed to provide a start point to construct the contig that spans the OsCem locus. The cloning and detailed functional analysis of the OsCem gene will help us to know more about the molecular mechanism of the embryo axis pattern and the Siamese polyembryo formation in rice.

In summary, our results suggest that the Siamese embryo with multiple plumules and/or multiple radicles in rice was regulated by a recessive $\mathrm{OsCem}$ gene, which might encode a protein related to auxin polar transport in the embryos and that is specifically expressed in embryo tissues and not in other vegetative tissues.

\section{Acknowledgements}

This work was, in part, supported by an international science foundation (IFS) grant, C/2741-1, and the University Research Initiative to (C-MH). We thank $\mathrm{J}$ Chen for critical reading of the article and the editor who corrected the grammar.

\section{References}

Benkova E, Michniewicz M, Sauer M, Teichmann T, Seifertova D, Jurgens G et al. (2003). Local, efflux-dependent auxin gradient as a common module for plant organ formation. Cell 115: 591-602.

Berleth T, Jurgens G (1993). The role of the monopteros gene in organising the basal body region of the Arabidopsis embryo. Development 118: 575-587.

Clark JK, Sheridan WF (1991). Isolation and characterization of 51 embryo-specific mutations in maize. Plant Cell 3: 935-951.

Erdelska O, Vidovencova Z (1992). Cleavage polyembryony in maize. Sex Plant Reprod 5: 224-226.

Errampalli D, Patton D, Castle L, Mickelson L, Hansen K, Schnall J et al. (1991). Embryonic lethals and T-DNA insertional mutagenesis in Arabidopsis. Plant Cell 3: 149-157.

Ferguson JD, McEwan JM, Card KA (1979). Hormonally induced polyembryos in wheat. Physiol Plant 45: 470-474.

Fischer C, Speth V, Fleig-Eberenz S, Neuhaus G (1997). Induction of zygotic polyembryos in wheat: influence of auxin polar transport. Plant Cell 9: 1767-1780.

Friml J, Vieten A, Sauer M, Weijers D, Schwartz H, Hamann T et al. (2003). Efflux-dependent auxin gradients establish the apical-basal axis of Arabidopsis. Nature 426: 147-153.

Heckel T, Werner K, Sheridan WF, Dumas C, Rogowsky PM (2000). Novel phenotype and developmental arrest in early embryo specific mutants of maize. Planta 210: 1-8.

Hong SK, Aoki T, Kitano H, Satoh H, Nagato Y (1995). Phenotypic diversity of 188 rice embryo mutants. Dev Genet 16: 298-310.

Hong SK, Kitano H, Satoh H, Nagato Y (1996). How is embryo size genetically regulated in rice. Development 122: 2051-2059.

Itoh J-I, Nonomura K-I, Ikeda K, Yamaki S, Inukai Y, Yamagishi $\mathrm{H}$ et al. (2005). Rice plant development: from zygote to spikelet. Plant Cell Physiol 46: 23-47.

Jurgens G (2001). Apical-basal pattern formation in Arabidopsis embryogenesis. EMBO J 14: 3609-3616. 
Kepinski S (2006). Integrating hormone signaling and patterning mechanisms in plant development. Curr Opin Plant Biol 9: 28-34.

Kinae T, Hong S-K, Nagato Y (2005). Apical displacement1 gene regulates apical-basal pattern formation in rice embryo. Plant Sci 168: 1345-1351.

Kitano H, Tamura Y, Satoh H, Nagato Y (1993). Hierarchical regulation of organ differentiation during embryogenesis in rice. Plant J 3: 607-610.

Lander ES, Green P, Abrahamson J, Barlow A, Daly MJ, Lincoln SE et al. (1987). MAPMAKER: an interactive computer package for constructing primary genetic linkage maps of experimental and natural populations. Genomics 1: 174-181.

Lukowitz W, Roeder A, Parmenter D, Somerville C (2004). A MAPKK kinase gene regulates extra-embryonic cell fate in Arabidopsis. Cell 116: 109-119.

Mayer U, Torres Ruiz RA, Berleth T, Misera S, Jurgens G (1991). Mutations affecting body organization in the Arabidopsis embryo. Nature 353: 402-407.

McCouch S, Kochert G, Yu ZH, Wang ZY, Khush GS, Coffman WR et al. (1988). Molecular mapping of rice chromosomes. Theor Appl Genet 76: 815-820.

Morgan DT, Rappleye RD (1951). Polyembryony in maize and lily following X-irradiation of the pollen. J Hered 42: 90-93.

Nagato Y, Kitano H, Kamijima O, Kikuchi S, Satoh H (1989) Developmental mutants showing abnormal organ differentiation in rice embryos. Theor Appl Genet 78: 11-15.

Reinhardt D, Pesce E-R, Stieger P, Mendel T, Baltensperger K, Bennett $M$ et al. (2003). Regulation of phyllotaxis by polar auxin transport. Nature 426: 255-260.
Sabatini S, Beis D, Wolkenfelt H, Murfeltt J, Guilfoyle T, Malamy J et al. (1999). An auxin-dependent distal organizater of pattern and polarity in the Arabidopsis root. Cell 99: 463-474.

Satoh N, Hong S-K, Nishimura A, Matsuoka M, Kitano H, Nagato Y (1999). Initiation of shoot apical meristem in rice: characterization of four SHOOTLESS genes. Development 126: 3629-3636.

Schmelz EA, Engelberth J, Alborn HT, O'Donnell P, Sammons M, Toshima $\mathrm{H}$ et al. (2003). Simultaneous analysis of phytohormones, phytotoxins, and volatile organic compounds in plants. Proc Natl Acad Sci USA 100: 10552-10557.

Schrick K, Mayer U, Horrichs A, Kuhnt C, Bellini C, Dangl J et al. (2000). FACKEL is a sterol C-14 reductase required for organized cell division and expansion in Arabidopsis embryogenesis. Genes Dev 14: 1471-1484.

Sheridan WF, Clark JK (1993). Mutational analysis of morphogenesis of the maize embryo. Plant J 3: 347-358.

Shimamoto K, Kyozuka J (2002). Rice as model for comparative genomics of plants. Annu Rev Plant Biol 53: 399-419.

Unrau J, Larter EN (1952). Cytogenetical response of cereals to 2,4-D. I. Study of meiosis of plants treated at various stages of growth. Can J Bot 30: 22-27.

Vernon DM, Meinke DW (1994). Embryogenic transformation of the suspensor in twin, a polyembryonic mutant of Arabidopsis. Dev Biol 165: 566-573.

Zhang JZ, Somerville CR (1997). Suspensor-derived polyembryony caused by altered expression of valyl-Trna synthetase in the twn2 mutant of Arabidopsis. Proc Natl Acad Sci USA 94: 7349-7355. 